\title{
SU(6), Triquark states, and the pentaquark
}

\author{
Swarup Kumar Majee* and Amitava Raychaudhuri \\ Harish-Chandra Research Institute, \\ Chhatnag Road, Jhunsi, Allahabad 211 019, India \\ and \\ Department of Physics, University of Calcutta, \\ 92 Acharya Prafulla Chandra Road, Kolkata 700 009, India
}

\begin{abstract}
The purported observation of a state $\Theta^{+}$with strangeness $\mathrm{S}=+1$ led to its quark model interpretation in terms of a pentaquark combination involving a triquark-diquark structure - the Karliner-Lipkin model. In this work, the proper colour-spin symmetry properties for the $q q \bar{q}$ triquark are elucidated by calculating the SU(6) unitary scalar factors and Racah coefficients. Using these results, the colour-spin hyperfine interactions, including flavour symmetry breaking therein, become straight-forward to incorporate and the pentaquark masses are readily obtained. We examine the effect on the pentaquark mass of (a) deviations from the flavour symmetric limit and (b) different strengths of the doublet and triplet hyperfine interactions. Reference values of these parameters yield a $\Theta^{+}$mass prediction of $1601 \mathrm{MeV}$ but it can comfortably accommodate $1540 \mathrm{MeV}$ for alternate choices. In the same framework, other pentaquark states $\Xi(\mathrm{S}=-2)$ and $\Theta^{c}$ (with charm $\mathrm{C}=-1$ ) are expected at $1783 \mathrm{MeV}$ and $2757 \mathrm{MeV}$, respectively.
\end{abstract}

PACS Nos.: 12.39.-x, 14.20.-c, 12.40.Yx, 12.38.-t

${ }^{*}$ E-mail address: swarup@mri.ernet.in 


\section{Introduction}

Since long, baryon spectroscopy has been an arena to learn about low-energy quantum chromodynamics. The purported observation of a narrow baryon state of strangeness +1 at a mass around $1540 \mathrm{MeV}, \Theta^{+}$, by several experiments [1 brought renewed attention to this theatre. The evidence in support of this new state is now of conflicting nature, loaded more in the direction of non-observation [2, 3]. Within the quark picture, the positive strangeness $(\equiv \bar{s})$ of the $\Theta^{+}$baryon puts it in an exotic category and entails an interpretation in terms of a minimum of four quarks and an antiquark - a pentaquark state $(u d u d \bar{s})$.

Soon after, three other states which also demand a pentaquark classification were also observed. These are the $\Xi^{--}(d s d s \bar{u})$ and $\Xi^{0}(d s u s \bar{d})$ both at $1862 \mathrm{MeV}$ [4] and the $\Sigma^{c}(u d u d \bar{c})$ [5] with mass $3099 \mathrm{MeV}$.

Though exotic states such as the pentaquark have a long history, particular attention was drawn to a possible $\Theta^{+}$-like state in the $\mathrm{SU}(3)$ version of the chiral soliton model 6]. Subsequently, the experimental results have stimulated the exploration of many ideas, e.g., quark clusters, colour hyperfine interactions, Goldstone boson exchange, QCD sum rules, lattice methods, etc., which have been reviewed in the literature [7]. For the $\Theta^{+}$, within the quark model framework, two models [8, 9] have achieved special prominence. It is convenient to discuss these using the language of $\mathrm{SU}(6)$ of colourspin, $\mathrm{SU}(3)$ of colour, and $\mathrm{SU}(2)$ of spin. Thus, for example, a quark transforms as $(6,3,2)$, where the three integers within the parentheses identify the representations of the above $\mathrm{SU}(6), \mathrm{SU}(3)$, and $\mathrm{SU}(2)$, respectively. To avoid cluttering, the flavour $\mathrm{SU}(3)$ structure is not explicitly shown. Our interest will be on the triquark state which is an ingredient of the Karliner-Lipkin model [8].

An alternative possibility is the Jaffe-Wilczek (JW) model [9]. Here the four quarks are assumed to form two diquark clusters, each in the $(21, \overline{3}, 1)$ representation. Of the four possible combinations for a two-quark cluster - $(21,6,3),(15,6,1),(15, \overline{3}, 3)$, $(21, \overline{3}, 1)$ - this is the one of the lowest energy. The two diquark clusters and the remaining antiquark - each one of which is in colour $\overline{3}$ - combine to form the colour singlet pentaquark state $(q q)(q q)(\bar{q})$, e.g., $\Theta^{+} \equiv(u d)(u d)(\bar{s})$. A relative orbital angular momentum, $\mathrm{L}=1$, is assumed between the diquarks; this is in tune with the observed narrow width of the state. Another consequence is that the pentaquark parity is predicted to be positive. Note that the colour-spin symmetric nature of the $(21, \overline{3}, 1)$ diquark requires it to be antisymmetric, $\overline{3}$, in flavour to satisfy the generalized Pauli principle. The two diquarks (colour $\overline{3}$ bosons) combine to form colour 3 to match up with the antiquark. This, and $\mathrm{L}=1$, requires the combination to be in a flavour

symmetric $\overline{6}$ state. The overall pentaquark flavour must be in $\overline{6} \otimes \overline{3}=8+\overline{10}$. The quantum numbers of $\Theta^{+}$can be accommodated only in the $\overline{10}$.

In the Karliner-Lipkin (KL) model the quark clustering is different. Here, it is postulated that there is one diquark cluster with the same quantum numbers as in the 
JW model. The difference is that the remaining two quarks and the antiquark are assumed to form a triquark cluster $(q q \bar{q})$ with the quantum numbers $(6,3,2)$ which is in a flavour $\overline{6}$. The pentaquark state is the colour singlet $(q q)(q q \bar{q})$ combination. To explain the narrowness of the observed states, a relative orbital angular momentum, $\mathrm{L}=1$, is postulated between the clusters so that the parity of the state is predicted to be positive in this model as well. The flavour structure of the states is the same as in the JW model.

In this work, we set two goals. First, we take a detailed look at the group theoretic properties of the triquark state. We derive expressions for the $\mathrm{SU}(6)$ unitary scalar factors and Racah coefficients related to the Clebsch-Gordan coefficients relevant for this state. Second, we use these results to estimate masses for pentaquark states. We indicate how flavour symmetry breaking may be incorporated in the analysis.

In the next section we present the $\mathrm{SU}(6)$ unitary scalar factors and Racah coefficients, which have been derived $a b$ initio. In section III we recall the nature of the colour-spin hyperfine interaction while in the following section we use it to estimate the hyperfine energies for baryons, mesons, diquarks, and triquarks. In section $\mathrm{V}$ the different threads are brought together for estimating pentaquark masses. In section VI we discuss the results. We end in section VII with our conclusions.

\section{Some group-theoretic results}

In this section, we collect some results about $\mathrm{SU}(6)$ unitary scalar factors and Racahlike coefficients which will be useful for the subsequent discussion. Though our motivation in obtaining these results is the triquark state, they may find some use in other applications of the $\mathrm{SU}(6)$ group.

\section{II.1 SU(6) unitary scalar factors}

To minimise the complexities, we first summarize the notations. A member of an $\mathrm{SU}(2)$ multiplet is denoted by $\left\{(2 I+1), I_{3}\right\} ;$ e.g., the $s_{z}=+\frac{1}{2}$ state of a spin-half particle is $\left\{2,+\frac{1}{2}\right\}$.

For $\mathrm{SU}(3)$, the sub-representations are designated by the $\mathrm{SU}(2)^{c}$ representation 11 and the 'hypercharge', $Y^{c}$. Thus, one uses the combination $\left\{R_{3}, \alpha, I_{3}^{c}\right\}$ where $R_{3}$ is the $\mathrm{SU}(3)$ representation and $\alpha \equiv\left[\left(2 I^{c}+1\right), Y^{c}\right]$. For illustration, a quark state with $I_{3}^{c}=+\frac{1}{2}$ and $Y^{c}=\frac{1}{3}$ will be denoted as $\left\{3,\left[2, \frac{1}{3}\right],+\frac{1}{2}\right\}$.

Putting the above together, an $\mathrm{SU}(6)$ state is denoted by $\left(R_{6},\left\{R_{3}, \alpha, I_{3}^{c}\right\},\left\{(2 I+1), I_{3}\right\}\right)$ where $R_{6}$ is the $\mathrm{SU}(6)$ representation while $\left\{R_{3}, \alpha, I_{3}^{c}\right\}$ and $\left\{(2 I+1), I_{3}\right\}$ characterize the corresponding $\mathrm{SU}(3)$ and $\mathrm{SU}(2)$ sub-representations. The quark state mentioned above, will be $\left(6,\left\{3,\left[2, \frac{1}{3}\right],+\frac{1}{2}\right\},\left\{2, \pm \frac{1}{2}\right\}\right)$, where the $\mathrm{SU}(3)(\mathrm{SU}(2))$ quantum numbers

\footnotetext{
${ }^{1}$ The superscript ' $c$ ' has been added to indicate the subgroups of $\mathrm{SU}(3)$.
} 
are enclosed in the first (second) braces. In most of the following, it will be possible to suppress $\alpha, I_{3}^{c}$ and $I_{3}-e . g$. , the quark state $\equiv(6,3,2)$. This is because the unitary scalar factors and the Racah coefficients are independent of $\alpha, I_{3}^{c}$ and $I_{3}$.

The SU(6) unitary scalar factors are generalisations of the SU(3) isoscalar factors. The Clebsch-Gordan (CG) coefficients of SU(2) are well known. If $i \otimes j=k \oplus \ldots$, where $i, j, k$ are $\mathrm{SU}(2)$ representations, we use $C G\left(S U(2)_{i, j, k}\right)$ as an abbreviation for the usual $C_{i_{3}, j_{3}, k_{3}}^{i, j, k}[10]$.

Using the $\mathrm{SU}(2)$ submultiplets within an $\mathrm{SU}(3)$ representation, the $\mathrm{CG}$ coefficients for $\mathrm{SU}(3)$ can be expressed in terms of products of isoscalar factors and $\mathrm{SU}(2) \mathrm{CG}$ coefficients. Schematically, for the case $P \otimes Q=R \oplus \ldots$ :

$$
C G\left(S U(3)_{P, Q, R}\right)=\left[\begin{array}{ccc}
P & Q & R \\
\alpha_{P} & \alpha_{Q} & \alpha_{R}
\end{array}\right] \times C G\left(S U(2)_{I_{P}, I_{Q}, I_{R}}\right)
$$

where the $\alpha_{i}, i=P, Q, R$ indicate the sub-representations of the $\mathrm{SU}(3)$ representations $P, Q, R$. The first factor on the right-hand-side is the $\mathrm{SU}(3)$ isoscalar factor. It is independent of $I_{P 3}, I_{Q 3}, I_{R 3}$. Tables of $\mathrm{SU}(3)$ isoscalar factors have been available for long [11].

Similarly, in $\mathrm{SU}(6)$, if $X \otimes Y=Z \oplus \ldots$ then

$$
\begin{aligned}
C G\left(S U(6)_{X, Y, Z}\right)= & {\left[\begin{array}{ccc}
X & Y & Z \\
\left(P_{X}, I_{X}\right) & \left(P_{Y}, I_{Y}\right) & \left(P_{Z}, I_{Z}\right)
\end{array}\right] } \\
& \times C G\left(S U(3)_{P_{X}, P_{Y}, P_{Z}}\right) \times C G\left(S U(2)_{I_{X}, I_{Y}, I_{Z}}\right) .
\end{aligned}
$$

Here, the first factor on the right-hand-side is an $\mathrm{SU}(6)$ unitary scalar factor - the generalization of the $\mathrm{SU}(3)$ isoscalar factor. $P_{X}\left(I_{X}\right)$ indicates the $\mathrm{SU}(3)$ (SU(2)) subrepresentation within the $\mathrm{SU}(6)$ multiplet $X$.

Since the triquark state is made out of two quarks $\left(q_{1}, q_{2}\right)$ and an antiquark $\left(\bar{q}_{3}\right)$, the following $\mathrm{SU}(6)$ combinations arise:

$$
\begin{gathered}
q q \text { state }: \quad 6 \otimes 6=21 \oplus 15 \\
q q \bar{q} \text { state }: \quad 21 \otimes \overline{6}=120 \oplus 6_{1}^{\phi}, \quad 15 \otimes \overline{6}=84 \oplus 6_{2}^{\phi} .
\end{gathered}
$$

or, alternatively,

$$
\begin{gathered}
q \bar{q} \text { state }: \quad 6 \otimes \overline{6}=35 \oplus 1 \\
q \bar{q} q \text { state }: \quad 35 \otimes 6=120 \oplus 84 \oplus 6_{1}^{\psi}, \quad 1 \otimes 6=6_{2}^{\psi} .
\end{gathered}
$$

The superscripts $\phi$ and $\psi$ will be clarified in the next subsection where we identify the Racah coefficients which relate $\left(6_{1}^{\phi}, 6_{2}^{\phi}\right)$ to $\left(6_{1}^{\psi}, 6_{2}^{\psi}\right)$.

For the purpose of the triquark, the $\mathrm{SU}(6) \mathrm{CG}$ coefficients for the product $21 \otimes \overline{6}=120$ $\oplus 6$ are necessary. We have not been able to find the SU(6) unitary scalar factors for 
this product in the published literature [12]. Here, therefore, their ab initio calculated values are presented. We follow the generalized Condon-Shortley phase convention [13] and obtain:

$$
\left[\begin{array}{ccc}
21 & \overline{6} & 6 \\
(6,3) & (\overline{3}, 2) & (3,2)
\end{array}\right]=\sqrt{\frac{6}{7}}, \quad\left[\begin{array}{ccc}
21 & \overline{6} & 6 \\
(\overline{3}, 1) & (\overline{3}, 2) & (3,2)
\end{array}\right]=\sqrt{\frac{1}{7}}
$$

Also,

$$
\left[\begin{array}{ccc}
21 & \overline{6} & 120 \\
(6,3) & (\overline{3}, 2) & (3,2)
\end{array}\right]=\sqrt{\frac{1}{7}}, \quad\left[\begin{array}{ccc}
21 & \overline{6} & 120 \\
(\overline{3}, 1) & (\overline{3}, 2) & (3,2)
\end{array}\right]=-\sqrt{\frac{6}{7}} .
$$

For the sake of completeness, the SU(6) unitary scalar factors for the case $15 \otimes \overline{6}=$ $84 \oplus 6$ are:

$$
\left[\begin{array}{ccc}
15 & \overline{6} & 6 \\
(6,1) & (\overline{3}, 2) & (3,2)
\end{array}\right]=\sqrt{\frac{2}{5}}, \quad\left[\begin{array}{ccc}
15 & \overline{6} & 6 \\
(\overline{3}, 3) & (\overline{3}, 2) & (3,2)
\end{array}\right]=\sqrt{\frac{3}{5}}
$$

and

$$
\left[\begin{array}{ccc}
15 & \overline{6} & 84 \\
(6,1) & (\overline{3}, 2) & (3,2)
\end{array}\right]=\sqrt{\frac{3}{5}}, \quad\left[\begin{array}{ccc}
15 & \overline{6} & 84 \\
(\overline{3}, 3) & (\overline{3}, 2) & (3,2)
\end{array}\right]=-\sqrt{\frac{2}{5}} .
$$

\section{II.2 Racah coefficients for the triquark cluster}

\section{II.2.1 SU(2) and SU(3)}

In this subsection, after recapitulating the concept of Racah coefficients, using angular momentum as an illustration, the necessary results useful for the triquark case are presented.

When three angular momenta $j_{1}, j_{2}, j_{3}$ are added, one can obtain the same final angular momentum $j$ by, for example, (a) combining $j_{1}$ and $j_{2}$ first to get $j_{12}$ and adding $j_{3}$ to it, or by (b) first adding $j_{1}$ and $j_{3}$ to obtain $j_{13}$ and then combining it with $j_{2}$, or by (c) adding $j_{2}$ and $j_{3}$ to obtain $j_{23}$ and then adding $j_{1}$ to it. The states of the representation $j$ obtained by these three different routes, may be denoted by $\left|j_{1}, j_{2}, j_{3} ; j_{12}, j, m\right\rangle$, $\left|j_{1}, j_{2}, j_{3} ; j_{13}, j, m\right\rangle$, and $\left|j_{1}, j_{2}, j_{3} ; j_{23}, j, m\right\rangle$, respectively. These three sets of states are related to each other by unitary transformations whose coefficients, $U$, are called the normalized Racah coefficients. For example,

$$
U\left(j_{1}, j_{2}, j_{3}, j ; j_{12}, j_{13}\right)=\left\langle j_{1}, j_{2}, j_{3} ; j_{12}, j, m \mid j_{1}, j_{2}, j_{3} ; j_{13}, j, m\right\rangle .
$$

The triquark state is of the structure $\left(q_{1} q_{2} \bar{q}_{3}\right)$. Since the quarks (antiquarks) transform as $6(\overline{6})$ of colour-spin $\mathrm{SU}(6)$, for the analysis of these states one requires the Racah coefficients for $\mathrm{SU}(6)$ for the product $6 \times 6 \times \overline{6}$. 
For most purposes, it actually suffices if one has the colour SU(3) and spin SU(2) Racah coefficients.

The same final triquark state may be reached by first combining $q_{1}$ and $q_{2}$ (colour: $3 \times 3=\overline{3}+6$ and spin: $2 \times 2=3+1$ ) and then combining with each of these possibilities the antiquark state $\bar{q}_{3}$. An alternate way of obtaining the same state is to first pair $q_{1}$ with $\bar{q}_{3}$ (colour: $3 \times \overline{3}=8+1$ and spin: $2 \times 2=3+1$ ) and then adjoining $q_{2}$ to the result. A third possibility is obtained by interchanging $q_{1} \leftrightarrow q_{2}$ in the previous alternative.

We concentrate, in the interest of the pentaquark application, on the triqark state which transforms like a colour $\mathrm{SU}(3)$ triplet and an $\mathrm{SU}(2)$ doublet. The basis states in this sector may be denoted as:

$$
\left(\begin{array}{c}
\left|\phi_{1}\right\rangle \\
\left|\phi_{2}\right\rangle \\
\left|\phi_{3}\right\rangle \\
\left|\phi_{4}\right\rangle
\end{array}\right) \equiv\left(\begin{array}{l}
\left|\left(q_{1} q_{2}\right)_{1}^{3}\left(\bar{q}_{3}\right)_{2}^{\overline{3}}\right\rangle_{(3,2)} \\
\left|\left(q_{1} q_{2}\right)_{1}^{6}\left(\bar{q}_{3}\right)_{2}^{3}\right\rangle_{(3,2)} \\
\left|\left(q_{1} q_{2}\right)_{3}^{3}\left(\bar{q}_{3}\right)_{2}^{3}\right\rangle_{(3,2)} \\
\left|\left(q_{1} q_{2}\right)_{3}^{6}\left(\bar{q}_{3}\right)_{2}^{3}\right\rangle_{(3,2)}
\end{array}\right)
$$

and

$$
\left(\begin{array}{c}
\left|\psi_{1}\right\rangle \\
\left|\psi_{2}\right\rangle \\
\left|\psi_{3}\right\rangle \\
\left|\psi_{4}\right\rangle
\end{array}\right) \equiv\left(\begin{array}{l}
\left|\left(q_{1} \bar{q}_{3}\right)_{1}^{1}\left(q_{2}\right)_{2}^{3}\right\rangle_{(3,2)} \\
\left|\left(q_{1} \bar{q}_{3}\right)_{1}^{8}\left(q_{2}\right)_{2}^{3}\right\rangle_{(3,2)} \\
\left|\left(q_{1} \bar{q}_{3}\right)_{3}^{1}\left(q_{2}\right)_{2}^{3}\right\rangle_{(3,2)} \\
\left|\left(q_{1} \bar{q}_{3}\right)_{3}^{8}\left(q_{2}\right)_{2}^{3}\right\rangle_{(3,2)}
\end{array}\right), \quad\left(\begin{array}{c}
\left|\chi_{1}\right\rangle \\
\left|\chi_{2}\right\rangle \\
\left|\chi_{3}\right\rangle \\
\left|\chi_{4}\right\rangle
\end{array}\right) \equiv\left(\begin{array}{c}
\left|\left(q_{2} \bar{q}_{3}\right)_{1}^{1}\left(q_{1}\right)_{2}^{3}\right\rangle_{(3,2)} \\
\left|\left(q_{2} \bar{q}_{3}\right)_{1}^{8}\left(q_{1}\right)_{2}^{3}\right\rangle_{(3,2)} \\
\left|\left(q_{2} \bar{q}_{3}\right)_{3}^{1}\left(q_{1}\right)_{2}^{3}\right\rangle_{(3,2)} \\
\left|\left(q_{2} \bar{q}_{3}\right)_{3}^{8}\left(q_{1}\right)_{2}^{3}\right\rangle_{(3,2)}
\end{array}\right)
$$

The notation used here, for example, is that the triquark state with $\mathrm{SU}(3)(\mathrm{SU}(2))$ multiplicity $c^{\prime}\left(s^{\prime}\right)$ obtained through the diquark combination $\left(q_{1} q_{2}\right)$ with $\mathrm{SU}(3)$ and $\mathrm{SU}(2)$ multiplicity $c$ and $s$, respectively, is represented as $\left|\left(q_{1} q_{2}\right)_{s}^{c}\left(\bar{q}_{3}\right)_{2}^{\overline{3}}\right\rangle_{\left(c^{\prime}, s^{\prime}\right)}$.

These possibilities are related by Racah-like coefficients which are found by explicit calculation to be:

$$
\left(\begin{array}{l}
\left|\phi_{1}\right\rangle \\
\left|\phi_{2}\right\rangle \\
\left|\phi_{3}\right\rangle \\
\left|\phi_{4}\right\rangle
\end{array}\right)=\left(\begin{array}{cccc}
-\frac{1}{2 \sqrt{3}} & \frac{1}{\sqrt{6}} & \frac{1}{2} & -\frac{1}{\sqrt{2}} \\
\frac{1}{\sqrt{6}} & \frac{1}{2 \sqrt{3}} & -\frac{1}{\sqrt{2}} & -\frac{1}{2} \\
\frac{1}{2} & -\frac{1}{\sqrt{2}} & \frac{1}{2 \sqrt{3}} & -\frac{1}{\sqrt{6}} \\
-\frac{1}{\sqrt{2}} & -\frac{1}{2} & -\frac{1}{\sqrt{6}} & -\frac{1}{2 \sqrt{3}}
\end{array}\right)\left(\begin{array}{l}
\left|\psi_{1}\right\rangle \\
\left|\psi_{2}\right\rangle \\
\left|\psi_{3}\right\rangle \\
\left|\psi_{4}\right\rangle
\end{array}\right)
$$

and

$$
\left(\begin{array}{l}
\left|\phi_{1}\right\rangle \\
\left|\phi_{2}\right\rangle \\
\left|\phi_{3}\right\rangle \\
\left|\phi_{4}\right\rangle
\end{array}\right)=\left(\begin{array}{cccc}
-\frac{1}{2 \sqrt{3}} & \frac{1}{\sqrt{6}} & \frac{1}{2} & -\frac{1}{\sqrt{2}} \\
-\frac{1}{\sqrt{6}} & -\frac{1}{2 \sqrt{3}} & \frac{1}{\sqrt{2}} & \frac{1}{2} \\
-\frac{1}{2} & \frac{1}{\sqrt{2}} & -\frac{1}{2 \sqrt{3}} & \frac{1}{\sqrt{6}} \\
-\frac{1}{\sqrt{2}} & -\frac{1}{2} & -\frac{1}{\sqrt{6}} & -\frac{1}{2 \sqrt{3}}
\end{array}\right)\left(\begin{array}{l}
\left|\chi_{1}\right\rangle \\
\left|\chi_{2}\right\rangle \\
\left|\chi_{3}\right\rangle \\
\left|\chi_{4}\right\rangle
\end{array}\right)
$$




\section{II.2.2 $\mathrm{SU}(6)$ Racah coefficients}

One can use the unitary scalar factors in eqs. (7) - (8) to write:

$$
\left|q_{1} q_{2} \bar{q}_{3}\right\rangle_{\left(6_{1}^{\phi}, 3,2\right)}=\sqrt{\frac{6}{7}}\left|\phi_{4}\right\rangle+\sqrt{\frac{1}{7}}\left|\phi_{1}\right\rangle, \quad\left|q_{1} q_{2} \bar{q}_{3}\right\rangle_{(120,3,2)}=\sqrt{\frac{1}{7}}\left|\phi_{4}\right\rangle-\sqrt{\frac{6}{7}}\left|\phi_{1}\right\rangle .
$$

From eqs. (9) - (10) the states obtained if the diquarks are in the 15 of $\mathrm{SU}(6)$ are:

$$
\left|q_{1} q_{2} \bar{q}_{3}\right\rangle_{\left(6_{2}^{\phi}, 3,2\right)}=\sqrt{\frac{2}{5}}\left|\phi_{2}\right\rangle+\sqrt{\frac{3}{5}}\left|\phi_{3}\right\rangle, \quad\left|q_{1} q_{2} \bar{q}_{3}\right\rangle_{(84,3,2)}=\sqrt{\frac{3}{5}}\left|\phi_{2}\right\rangle-\sqrt{\frac{2}{5}}\left|\phi_{3}\right\rangle .
$$

Using eq. (14) one then has:

$$
\left|q_{1} q_{2} \bar{q}_{3}\right\rangle_{\left(6_{1}^{\phi}, 3,2\right)}=-\sqrt{\frac{7}{12}}\left|\psi_{1}\right\rangle-\sqrt{\frac{2}{21}}\left|\psi_{2}\right\rangle-\sqrt{\frac{1}{28}}\left|\psi_{3}\right\rangle-\sqrt{\frac{2}{7}}\left|\psi_{4}\right\rangle
$$

and

$$
\left|q_{1} q_{2} \bar{q}_{3}\right\rangle_{\left(6_{2}^{\phi}, 3,2\right)}=\sqrt{\frac{5}{12}}\left|\psi_{1}\right\rangle-\sqrt{\frac{2}{15}}\left|\psi_{2}\right\rangle-\sqrt{\frac{1}{20}}\left|\psi_{3}\right\rangle-\sqrt{\frac{2}{5}}\left|\psi_{4}\right\rangle
$$

Thus, one arrives at the Racah coefficients:

$$
\left(\begin{array}{l}
\left|\left(6_{1}^{\phi}, 3,2\right)\right\rangle \\
\left|\left(6_{2}^{\phi}, 3,2\right)\right\rangle
\end{array}\right)=\left(\begin{array}{cc}
\sqrt{\frac{5}{12}} & -\sqrt{\frac{7}{12}} \\
\sqrt{\frac{7}{12}} & \sqrt{\frac{5}{12}}
\end{array}\right)\left(\begin{array}{l}
\left|\left(6_{1}^{\psi}, 3,2\right)\right\rangle \\
\left|\left(6_{2}^{\psi}, 3,2\right)\right\rangle
\end{array}\right)
$$

The non-trivial unitary scalar factors corresponding to eq. (6) can be written as:

$$
\left[\begin{array}{ccc}
35 & 6 & \alpha \\
i & (3,2) & (3,2)
\end{array}\right]=U_{i, \alpha}
$$

with $i=1,2,3$ corresponding to $(8,1),(1,3)$, and $(8,3)$ while $\alpha=1,2,3$ to 120,84 , and $6_{1}^{\psi}$. Then,

$$
U=\left(\begin{array}{ccc}
-\sqrt{\frac{9}{28}} & -\sqrt{\frac{8}{21}} & \sqrt{\frac{25}{84}} \\
\sqrt{\frac{9}{20}} & -\sqrt{\frac{8}{15}} & -\sqrt{\frac{1}{60}} \\
-\sqrt{\frac{8}{35}} & -\sqrt{\frac{3}{35}} & -\sqrt{\frac{24}{35}}
\end{array}\right),
$$

Now we turn to the application of these results to the pentaquark. 


\section{Colour-spin hyperfine interaction}

Besides colour electric forces between all quarks and antiquarks, there exists a colourspin hyperfine (colour magnetic) interaction [14]. In the KL model, it is assumed that this interaction is operative inside the clusters but, due to the larger separation, the hyperfine interaction between clusters is negligible2. The colour-spin SU(6) hyperfine interaction energy is:

$$
V=-\sum_{i>j} v_{i j}\left(\overrightarrow{\sigma_{i}} \cdot \overrightarrow{\sigma_{j}}\right)\left(\overrightarrow{\lambda_{i}} \cdot \overrightarrow{\lambda_{j}}\right)
$$

Here, $\vec{\sigma}$ and $\vec{\lambda}$ are the Pauli and Gell-Mann matrices, and $i$ and $j$ run over the constituent quarks and antiquarks. The common practice is to take $v_{i j} \equiv v$ (flavour symmetry). $v$ captures information about the radial dependence of the bound state wave-function. For a composite system of $n_{q}$ quarks and $n_{\bar{q}}$ antiquarks, the hyperfine energy contribution is given by:

$$
E_{\text {hyp }}=\left[D(q+\bar{q})-2 D(q)-2 D(\bar{q})+16\left(n_{q}+n_{\bar{q}}\right)\right] v / 2,
$$

where

$$
D\left(R_{6}, R_{3}, s\right)=C_{6}\left(R_{6}\right)-C_{3}\left(R_{3}\right)-\frac{8}{3} s(s+1) .
$$

$C_{6}$ and $C_{3}$ are the quadratic Casimir operators of $\mathrm{SU}(6)$ and $\mathrm{SU}(3)$ respectively, and $s$, is the spin of the state. The effect of this hyperfine interaction on multiquark exotic states has been a topic of research over several decades [16, 17].

The mass estimate for the pentaquark proceeds along the following pattern. There are three contributions: (a) the masses of the constituent quarks, (b) the colour-spin hyperfine energy, and (c) the energy due to the P-wave excitation. The practice has been to estimate (a) from the masses of the decay products, (baryon + meson), since their quark content is the same as that of the parent; but here the hyperfine interaction contribution to the baryon and meson mass must be first subtracted out, as detailed in section V. Thus, the hyperfine interaction enters directly in (b) and also indirectly in (a) through the way it is extracted.

\section{Hyperfine energies}

\section{IV.1 Mesons and Baryons}

As noted, the hyperfine interaction contributions to the meson $(q \bar{q})$ and baryon $(q q q)$ masses are required for the estimation of the pentaquark mass. These can be readily

\footnotetext{
${ }^{2}$ Inclusion of the inter-cluster hyperfine interaction has also been considered [15].
} 
calculated using eq. (24). For example, in the flavour symmetry limit, one finds:

$$
E_{N(70,1,2)}=-8 v, \quad E_{\Delta(20,1,4)}=8 v, \quad E_{\pi(1,1,1)}=-16 v, \quad E_{\rho(35,1,3)}=\frac{16}{3} v,
$$

where in the parentheses the $\mathrm{SU}(6), \mathrm{SU}(3)$, and $\mathrm{SU}(2)$ properties of the particle have been indicated.

\section{IV.2 The diquark cluster}

As already mentioned, the diquark $(q q)$ is usually chosen to be in the $(21, \overline{3}, 1)$ representation which is symmetric in $\mathrm{SU}(6)$. In addition, a diquark can be in the $(21,6,3)$, $(15,6,1)$, and $(15, \overline{3}, 3)$ but these have higher energy. One finds from eq. (24) that the hyperfine energies for these four states are:

$$
E_{(21, \overline{3}, 1)}=-8 v, \quad E_{(21,6,3)}=-\frac{4}{3} v, \quad E_{(15,6,1)}=4 v, \quad E_{(15, \overline{3}, 3)}=\frac{8}{3} v .
$$

\section{IV.3 The triquark cluster}

The triquark cluster in the Karliner-Lipkin model is a member of the $(6,3,2)$ multiplet and contains two quarks and an antiquark. The two quarks are assumed to combine to a symmetric 21 of colour-spin $\mathrm{SU}(6)$. For $\mathrm{SU}(6) 21 \otimes \overline{6}=6 \oplus 120$, and the triquark $(120,3,2)$ carries higher hyperfine energy. If the two quarks are combined in an antisymmetric fashion, producing a 15 of $\mathrm{SU}(6)$, then 3 the triquark can be in $(6,3,2)$ or $(84,3,2)$.

More important is the fact that in the existing literature, the triquark in the $(6,3,2)$ is assumed to be made with the two quarks within the cluster forming a $(21,6,3)$. In actuality, so long as flavour symmetry of the hyperfine interaction holds, the lowest energy eigenstate of $\mathrm{SU}(6)$ receives contributions from both the $(21,6,3)$ and the $(21, \overline{3}, 1)$ combinations - see eq. (7) - and this triquark has the form given in the first expression in eq. (16). The other possible triquark states are the second expression in eq. (16) and the ones in eq. (17).

\section{IV.3.1 The triquark hyperfine energy}

The calculation of the triquark hyperfine energy using eq. (24) is complicated by the fact that the operator $D(q+\bar{q})$ and $D(q)$ do not commute; e.g., in eq. (16) an eigenstate of $D(q+\bar{q})$ is expressed as a linear combination of those of $D(q)$.

\footnotetext{
${ }^{3} \mathrm{In} \mathrm{SU}(6), 15 \otimes \overline{6}=6 \oplus 84$. In the absence of flavour symmetry, the triquark is a superposition of these and the 6 and 120 (see later).
} 
To circumvent this difficulty, we use the following procedure. We consider the contribution of eq. (23) for the triquark state term by term as:

$$
V=V_{12}\left(\overrightarrow{\sigma_{1}} \cdot \overrightarrow{\sigma_{2}}\right)\left(\overrightarrow{\lambda_{1}} \cdot \overrightarrow{\lambda_{2}}\right)+V_{13}\left(\overrightarrow{\sigma_{1}} \cdot \overrightarrow{\sigma_{3}}\right)\left(\overrightarrow{\lambda_{1}} \cdot \overrightarrow{\lambda_{3}}\right)+V_{23}\left(\overrightarrow{\sigma_{2}} \cdot \overrightarrow{\sigma_{3}}\right)\left(\overrightarrow{\lambda_{2}} \cdot \overrightarrow{\lambda_{3}}\right)
$$

The hyperfine energy from each term is most readily calculated in the basis where the two contributing quarks/antiquarks are first combined [18]; i.e., corresponding to the three terms in the r.h.s. of eq. (28) these are the $|\phi\rangle,|\psi\rangle$, and $|\chi\rangle$ bases of Sec. III, respectively. They are related to each other through eqs. (14) and (15). In terms of these basis states, one can immediately write down the expectation value of the Hamiltonian in eq. (28). Thus 4 , one has:

$$
\langle\phi|V| \phi\rangle=\left(\begin{array}{cccc}
\frac{4}{3} V_{12}+\frac{20}{3} V_{+}^{\phi} & 4 \sqrt{2} V_{-}^{\phi} & \frac{10}{\sqrt{3}} V_{-}^{\phi} & 2 \sqrt{6} V_{+}^{\phi} \\
4 \sqrt{2} V_{-}^{\phi} & -\frac{8}{3} V_{12}+\frac{8}{3} V_{+}^{\phi} & 2 \sqrt{6} V_{+}^{\phi} & \frac{4}{\sqrt{3}} V_{-}^{\phi} \\
\frac{10}{\sqrt{3}} V_{-}^{\phi} & 2 \sqrt{6} V_{+}^{\phi} & -4 V_{12} & 0 \\
2 \sqrt{6} V_{+}^{\phi} & \frac{4}{\sqrt{3}} V_{-}^{\phi} & 0 & 8 V_{12}
\end{array}\right),
$$

where $V_{ \pm}^{\phi}=V_{13} \pm V_{23}$. Analogously,

$$
\langle\psi|V| \psi\rangle=\left(\begin{array}{cccc}
\frac{8}{3} V_{12}+\frac{2}{3} V_{13}+\frac{28}{3} V_{23} & \frac{16}{3 \sqrt{2}} V_{-}^{\psi} & \frac{4}{\sqrt{3}} V_{12}-\frac{14}{\sqrt{3}} V_{23} & \frac{8}{\sqrt{6}} V_{+}^{\psi} \\
\frac{16}{3 \sqrt{2}} V_{-}^{\psi} & -\frac{16}{3} V_{13} & \frac{8}{\sqrt{6}} V_{+}^{\psi} & 0 \\
\frac{4}{\sqrt{3}} V_{12}-\frac{14}{\sqrt{3}} V_{23} & \frac{8}{\sqrt{6}} V_{+}^{\psi} & -2 V_{13} & 0 \\
\frac{8}{\sqrt{6}} V_{+}^{\psi} & 0 & 0 & 16 V_{13}
\end{array}\right)
$$

where $V_{ \pm}^{\psi}=V_{12} \pm V_{23} .\langle\chi|V| \chi\rangle$ is similar and is not presented here.

The eigenvalues and eigenvectors of this matrix give the triquark energy and its corresponding group theoretic configuration, respectively.

The method which we follow can be smoothly adopted to the case of flavour symmetry violation by appropriately changing the individual coupling strengths in the three terms of eq. (28). In the flavour symmetry limit, $V_{12}=V_{23}=V_{13}=v$, whence $V_{-}^{\phi}=V_{-}^{\psi}=0$. It is seen from eq. (29) that $\left(\phi_{1}, \phi_{4}\right)$ decouple from $\left(\phi_{2}, \phi_{3}\right)$ in this limit.

\section{Pentaquark masses}

\section{V.1 Hyperfine interaction couplings}

Needless to say, the strength of the colour-spin hyperfine interaction, $v$, is an important ingredient of the pentaquark mass estimation. The procedure has generally been to

\footnotetext{
${ }^{4}$ This form was noted in 18 .
} 
assume that it takes a universal value which is estimated by ascribing the $\Delta-N$ mass splitting to this interaction. Using eq. (26),

$$
v_{3}=\frac{m_{\Delta}-m_{N}}{16} \simeq 18.3 \mathrm{MeV}
$$

While this can be a first approximation, it should be borne in mind that $v$ is determined by the radial dependence of the bound state wave-function and thus is most likely different for two-body and three-body bound states. Indeed, using eq. (26) for the meson sector one has,

$$
v_{2}=\frac{m_{\rho}-m_{\pi}}{64 / 3} \simeq 29.6 \mathrm{MeV}
$$

This is actually an overestimate of $v_{2}$ since it is well known that the pion mass is too small for a simple quark model interpretation. Eq. (32) is only for the purpose of illustration 5 . However, it does indicate that it may not be unreasonable to expect that $v_{2} \neq v_{3}$ would give a better approximation to reality. In the following, in addition to discussing the results for the choice $v_{2}=v_{3}$, for the sake of comparison, we also use a $v_{2}$ for the diquarks different from the $v_{3}$ for the triquarks.

\section{V.2 Flavour symmetry breaking}

In the limit of exact flavour symmetry, the splitting between the lowest lying pseudoscalar mesons and the corresponding vector mesons with the same quark content would be flavour independent. A measure of flavour symmetry breaking can be obtained from

$$
x_{f}=\frac{m_{K^{*}}-m_{K}}{m_{\rho}-m_{\pi}} \simeq 0.63 .
$$

This suggests that the hyperfine interaction involving an $s$-quark or antiquark carries a suppression by the factor $x_{f}$. In eqs. (32) and (33) the use of $m_{\pi}$ makes the precise values inaccurate. To improve upon this, we use the masses of the heavier mesons $\rho, \phi, K^{*}$, and $K$. Using eq. (26), the hyperfine contributions for these states are, respectively,

$$
E_{\rho}=\frac{16}{3} v_{2}, \quad E_{\phi}=\frac{16}{3} x_{f 2}^{2} v_{2}, \quad E_{K^{*}}=\frac{16}{3} x_{f 2} v_{2}, \quad E_{K}=-16 x_{f 2} v_{2},
$$

Here we have added a subscript to $v$ and $x_{f}$ to indicate that these values of the hyperfine parameters apply for two-quark and/or antiquark systems. Using the masses of the mesons, one can solve for the hyperfine interaction parameters $\left(v_{2}, x_{f 2}\right)$ as well as the quark masses. In this manner, one gets:

$$
v_{2}=23.62 \mathrm{MeV}, x_{f 2}=0.782, m_{u, d}=322 \mathrm{MeV}, m_{s}=471 \mathrm{MeV} .
$$

\footnotetext{
${ }^{5}$ We extract $v_{2}$ from heavier mesons in the next subsection.
} 
These values are used in our subsequent calculations.

There are two three-body systems which enter in this analysis. One is the triquark state and the other the baryon to which the pentaquark decays. Just as for mesons, one can estimate the values of $v_{3}$ and $x_{f 3}$ from the $N-\Delta$ and $\Sigma-\Sigma^{*}$ mass splittings which are given by:

$$
E_{\Delta}-E_{N}=16 v_{3}, \quad E_{\Sigma^{*}}-E_{\Sigma}=\frac{16}{3} v_{3}\left(2 x_{f 3}+1\right), \quad E_{\Xi^{*}}-E_{\Xi}=\frac{16}{3} v_{3} x_{f 3}\left(x_{f 3}+2\right) .
$$

As a consistency check, we use the values so obtained to calculate the $\Xi-\Xi^{*}$ splitting and find that the agreement is not satisfactory. Therefore, we use all of the three above splittings to arrive at the best-fit values:

$$
v_{3}=17.89 \mathrm{MeV}, x_{f 3}=0.708 \text {. }
$$

In the following, these have been used for the triquark and baryons.

\section{V.3 P-wave excitation}

The energy due to the P-wave excitation can be estimated from the recently observed $D_{s}^{*}$ state at $2317 \mathrm{MeV}$, which is believed to be an orbital excitation of the state at 2112 $\mathrm{MeV}$. This gives

$$
E_{P}=m_{D_{s}^{*}}(P)-m_{D_{s}^{*}}(S) \simeq(2317-2112) \mathrm{MeV}=205 \mathrm{MeV} .
$$

\section{Results}

\section{VI.1 The flavour antidecuplet and the octet}

Putting together the inputs from the previous sections, one can readily obtain the masses of the pentaquark states in the Karliner-Lipkin model. For example, for $\Theta^{+}$, using eqs. (26) and (27):

$$
m_{\Theta^{+}}=\left\{\left(m_{N}+8 v_{3}\right)+\left(m_{s}+m_{q}\right)\right\}+E_{P}-8 v_{2}+E_{t r i}\left(v_{3}, x_{f 3}\right),
$$

where the expression in the curly brackets is the contribution from the quark masses. The last (penultimate) term is the hyperfine energy of the triquark (diquark). For other pentaquarks, the r.h.s. in eq. (39) has to be appropriately modified to reflect the quark content of the state and, when necessary, deviations from flavour symmetry have to be incorporated in eq. (28) to obtain the correct $E_{t r i}\left(v_{3}, x_{f 3}\right)$.

\footnotetext{
${ }^{6}$ Alternatively, one might use $E_{P}=m_{\Lambda\left(\frac{1}{2}\right)^{-}}-m_{\Lambda\left(\frac{1}{2}\right)^{+}} \simeq(1406-1116) \mathrm{MeV}=290 \mathrm{MeV}$. This will increase all pentaquark mass estimates below by $\sim 85 \mathrm{MeV}$.
} 


\begin{tabular}{|c|c|c|c|c|c|c|c|}
\hline \multirow{2}{*}{$\begin{array}{c}\text { Pentaquark } \\
\text { states }\end{array}$} & \multicolumn{7}{|c|}{ Mass (in MeV) } \\
\cline { 2 - 8 } & $\Theta^{+}$ & $N_{10}$ & $\Sigma_{10}$ & $\Xi_{10}$ & $N_{8}$ & $\Sigma_{8}$ & $\Xi_{8}$ \\
\hline Lowest & 1601 & 1358 & 1626 & 1783 & 2057 & 2217 & 2326 \\
\hline SU(6) Excited & 1789 & 1573 & 1840 & 1966 & 2321 & 2439 & 2512 \\
\hline
\end{tabular}

Table 1: Pentaquark lowest and first colour-spin excited state masses for the reference values of the parameters in eqs. (35) and (37).

As noted earlier, the pentaquark states fill an octet and an antidecuplet of flavour. Excepting for the three states, $\Theta^{+} \equiv u d u d \bar{s}, \Xi^{--} \equiv d s d s \bar{u}$, and $\Xi^{+} \equiv u s u s \bar{d}$, all other states in the antidecuplet have partners in the octet with identical isospin and hypercharge. In estimating the masses, we have assumed ideal mixing between the partners and ascribed the lighter member to the antidecuplet. Note that isospin symmetry is assumed unbroken, so it is enough to present the mass of one member of an isomultiplet. The masses of the pentaquark states at the reference values of the parameters see eqs. (35) and (37) - are given in Table 1.

In Fig. 1, in the left panel the antidecuplet pentaquark masses are shown as a function of the flavour symmetry violation parameter $x_{f}$, which assumes the value unity in the symmetry limit. In view of the closeness of the estimates of $x_{f}$ in eqs. (35) and (37), for this figure we have taken $x_{f 3}=x_{f 2}=x_{f}$. The triquark interaction strength has been kept fixed at $v_{3}=17.89 \mathrm{MeV}$. The bands arise from a variation of the strength of the diquark hyperfine interaction, $v_{2}$, with the lower edge corresponding to $v_{2}=v_{3}$ and the upper to $v_{2}=23.62 \mathrm{MeV}$ (see eq. (35)). For this figure, $E_{P}$ has been chosen as $209 \mathrm{MeV}$, following eq. (38). It is observed that the triquark corresponding to the lowest eigenvalue of the hyperfine energy Hamiltonian - eq. (29) - is predominantly a combination of the states $\phi_{1}$ and $\phi_{4}$ (see eq. (12)) which are antisymmetric in the quark flavours.

Note that, $N_{10}$, the non-strange member of the antidecuplet 7 is predicted to be at a mass of $1355 \mathrm{MeV}$ for $v_{2}=v_{3}$ which is enhanced to $\sim 1400 \mathrm{MeV}$ when $v_{2}=23.62 \mathrm{MeV}$ is used. This prediction is independent of the choice of $x_{f}$ since the state does not have strange quarks. For the exotic $\Xi_{10}^{--}$state the mass prediction is in the range $1795-$ $1825 \mathrm{MeV}$ for $x_{f}=0.7$ to be compared with that of the experimentally observed state at $1862 \mathrm{MeV}$ [4].

In the right panel of Fig. 1 are shown the octet pentaquark masses. The splitting between the masses of the octet states and the corresponding antidecuplet states is seen to be typically around 500-600 MeV. As noted earlier, at the level of these calculations, the masses of the $\mathrm{I}=1$ and $\mathrm{I}=0$ members of the octet with $\mathrm{S}=-1$ are the same. The

\footnotetext{
${ }^{7}$ This state could have been proposed as a possible interpretation of the Roper resonance at 1440 $\mathrm{MeV}$.
} 

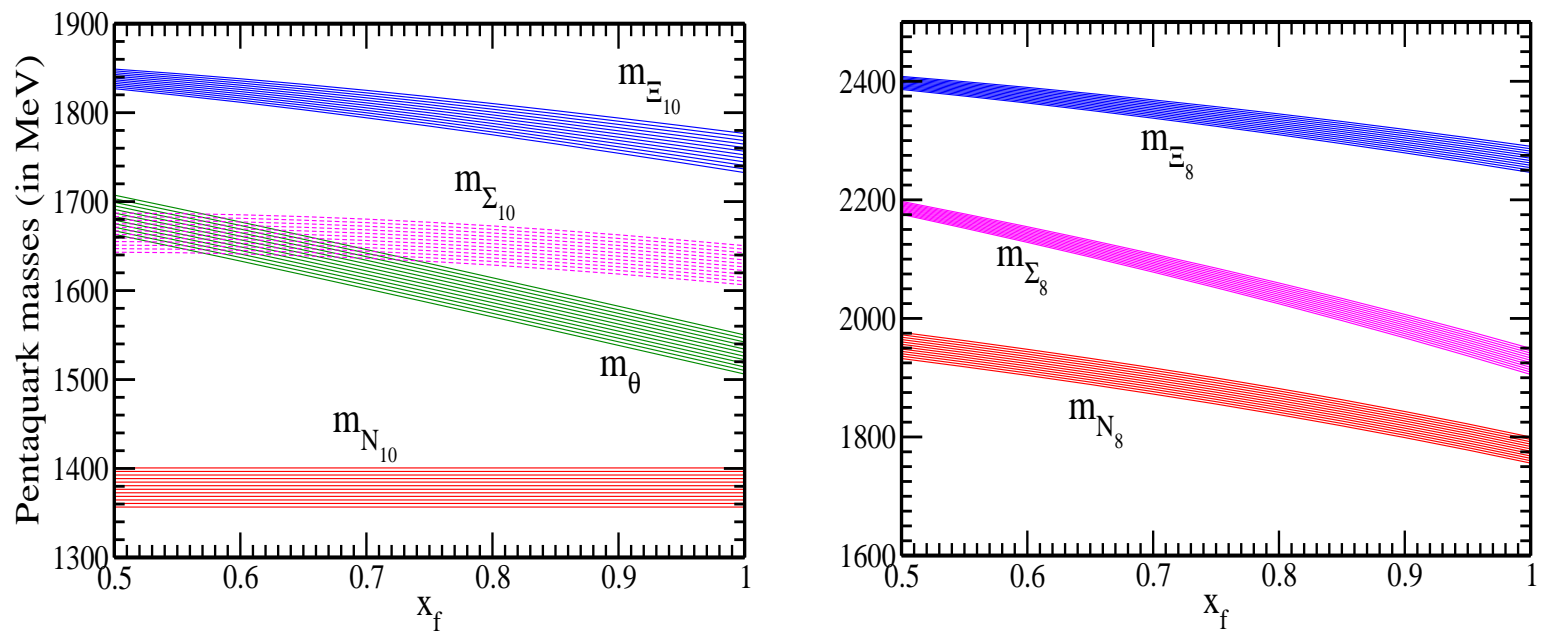

Figure 1: The dependence of pentaquark masses on the deviation from flavour symmetry $\left(x_{f}=1\right)$. The left (right) panel corresponds to flavour antidecuplet (octet) pentaquarks. The bands are obtained when the diquark hyperfine interaction strength is varied over the range $17.89 \mathrm{MeV} \leq v_{2} \leq 23.62 \mathrm{MeV}$ (see text).

non-strange neutral state in the octet, $N_{8}^{0}$, has the quark structure $(u d \bar{s})(d s)$ and its mass is consequently dependent on $x_{f}$.

A remark needs to be made about the symmetry property of the triquark state for the octet pentaquarks. This feature is most easily brought out from a consideration of the $\mathrm{S}=-2$ member of the octet, $\Xi_{8}$, which has the quark structure $(u s)(s s \bar{s})$. The diquark is antisymmetric in flavour so its choice is fixed. Unlike all the other states, here the triquark is compelled to have two identical $(s)$ quarks, besides the antiquark. Consequently, in the notation of section II, it can arise only from a combination of the states $\phi_{2}$ and $\phi_{3}$ (see eq. (12) ) which are symmetric in flavour. Obviously, all states in the pentaquark octet will share this feature in the exact flavour $\mathrm{SU}(3)$ limit.

The H1 experiment at HERA found evidence of a possible charmed pentaquark at mass $3099 \mathrm{MeV}$ [5]. This state has the quantum numbers of a pentaquark with the structure $u d u d \bar{c}$. Including flavour violation $\left(x_{f}=0.23\right.$ for the $\bar{c}$ quark $)$ and taking $v_{2}=23.62$ $\mathrm{MeV}, v_{3}=17.89 \mathrm{MeV}$, we find the predicted mass for such a state is $2757 \mathrm{MeV}$.

\section{VI.2 Triquark SU(6) excitations}

Colour triplet, spin $\frac{1}{2}$ triquarks come in four varieties. These are the four eigenstates of the hyperfine energy matrix in eq. (29). The results presented so far are obtained using the eigenstate with the minimum energy consistent with symmetry requirements 
- a certain choice of colour-spin assignments for the quark clusters - and leads to the lowest lying pentaquarks. It is evident that the other triquark eigenstate clusters also

lead to colour singlet spin $\frac{1}{2}$ pentaquark states, albeit heavier. How different are the masses in these other cases?

For illustration, we show in Table 1 the masses of the first excited partners of the antidecuplet and octet pentaquarks for the reference values of the hyperfine interaction parameters. In the flavour symmetry limit $\left(x_{f 3}=x_{f 2}=1\right)$, the spacing between the excited states is independent of the flavour and the lowest and first excited states are separated by $215 \mathrm{MeV}$ (370 MeV) for every member of the antidecuplet (octet).

There is no obvious argument to suppress the production of these additional states. It will be of interest to extend the ongoing searches to look for such SU(6) colour-spin excited partners, a novelty of QCD and the pentaquark system.

\section{Conclusions}

A pentaquark interpretation of the $\Theta^{+}$leads to predictions of several other colour singlet states in a similar mass range which populate an antidecuplet and an octet of flavour SU(3). In this paper, the masses of these pentaquark states have been calculated in a triquark-diquark (Karliner-Lipkin) model with refined estimates, upto first order, of the colour-spin SU(6) hyperfine interaction contributions.

Motivated by the structure of these states, the SU(6) unitary scalar factors relevant for the $q q \bar{q}$ triquark structure and the Racah coefficients, not available in the literature, have been calculated $a b$ initio. Using these results, the colour-spin $\mathrm{SU}(6)$ hyperfine contributions have been obtained taking two variations from the simplest picture. One of these concerns the deviation from flavour symmetry. The other originates from a possible difference in the strength of the hyperfine interaction for two- and three-quark bound states which can be related to the known splittings in baryonic and mesonic systems. Both of these variations do affect the pentaquark mass predictions. An element of uncertainty is introduced in these mass estimates by the P-wave excitation energy for which we have used the information from the $D$-meson system.

The triquark states within the antidecuplet and the octet are chosen, for good reason, to be the lowest eigenstate of the hyperfine energy Hamiltonian satisfying symmetry requirements. The other eigenstates are possible triquark states of SU(6) colour-spin excitations. The masses of colour singlet, spin $\frac{1}{2}$ pentaquarks resulting from these triquark excitations have also been estimated.

Irrespective of whether the claimed observation of the $\Theta^{+}$baryon is vindicated or not, pentaquarks can prove to be the tip of a revealing iceberg of new hadronic states illuminating novel facets of QCD. 


\section{Acknowledgements}

In the initial stages, this work was partially supported by the Department of Science and Technology, India (AR) and the Council of Scientific and Industrial Research, India (SKM).

\section{References}

[1] T. Nakano et al., (LEPS collaboration), Phys. Rev. Lett. 91, 012002 (2003);

V. V. Barmin et al., (DIANA Collaboration), Phys. At. Nuclei 66, 1715 (2003); ibid. 70, 35 (2007);

S. Stepanyan et al., (CLAS collaboration), Phys. Rev. Lett. 91, 252001 (2003); V. Kubarovsky et al., (CLAS collaboration), ibid. 92, 032001 (2004); erratum ibid 92, 049902 (2004);

J. Barth et al., (SAPHIR Collaboration), Phys. Lett. B572, 127 (2003);

A. Airpapetian et al., (HERMES Collaboration), ibid. B585, 213 (2004);

A. Aleev et al., (SVD Collaboration), Phys. At. Nuclei 68, 974 (2005); arXiv:hep-ex/0509033;

M. Abdel Bary, et al., (COSY-TOF Collaboration), Phys. Lett. B595, 127 (2004).

[2] M. Battaglieri et al., (CLAS Collaboration), Phys. Rev. Lett. 96, 042001 (2006); B. McKinnon et al., (CLAS Collaboration), ibid. 96, 212001 (2006); D. G. Ireland et al., (CLAS Collaboration), arXiv:0709.3154 [hep-ph].

[3] For reviews, see, for example, J. Pochodzalla, arXiv:hep-ex/0406077; M. Danilov and R. Mizuk, arXiv:0704.3531 [hep-ex]; K. Hicks, arXiv:hep-ex/0703004.

[4] C. Alt et al., (NA49 collaboration), Phys. Rev. Lett. 92, 042003 (2004); see, however, I. Abt et al., (HERA-B collaboration), ibid. 93, 212003 (2004); J. M. Link et al., (FOCUS collaboration), arXiv:0708.1010 [hep-ex].

[5] A. Aktas et al., (H1 Collaboration), Phys. Lett. B588, 17 (2004); hep-ex/0403017; see, however, S. Chekanov et al., (ZEUS Collaboration), Eur. Phys. J. C38, 29 (2004).

[6] Work on the chiral soliton model can be traced from D. Diakonov, V. Petrov, and M. Polyakov, Z. Physik A359, 305 (1997); A.V. Manohar, Nucl. Phys. B248, 19 (1984); M. Chemtob, Nucl. Phys. B256, 600 (1985); L.C. Biedenharn and Y. Dothan, in From SU(3) to Gravity, edited by E. Gotsman and G. Tauber, Cambridge University Press, Cambridge (1985); F, H. Walliser and H. Weigel, Eur. Phys. J. A26, 361 (2005). 
[7] For an overall picture, see, for example, B. K. Jennings and K. Maltman, Phys. Rev. D69, 094020 (2004); R.L. Jaffe, Phys. Rept. 409, 1 (2005).

[8] M. Karliner and H. J. Lipkin, arXiv:hep-ph/0307243; Phys. Lett. B575, 249 (2003).

[9] R. L. Jaffe and F. Wilczek, Phys. Rev. Lett. 91, 232003 (2003).

[10] E. U. Condon and H. Odabaşi, Atomic Structure, Cambridge University Press, Cambridge (1980).

[11] J. J. de Swart, Rev. Mod. Phys. 35, 916 (1963).

[12] See, for example, C. L. Cook and G. Murtaza, Nuovo Cim. 34, 531 (1965); R. H. Capps, Phys. Rev. Lett. 14, 31 (1965); M. Machacek and Y. Tomozawa, J. Math. Phys. 17, 458 (1976).

[13] G. E. Baird and L. C. Biedenharn, J. Math. Phys. 4, 1449 (1963); D. B. Lichtenberg, Unitary Symmetries and Elementary Particles, 2nd Ed., Academic Press, New York (1978).

[14] A. De Rujula, H. Georgi, and S. L. Glashow, Phys. Rev. D12, 147 (1975).

[15] K. Cheung, Phys. Rev. D69, 094029 (2004).

[16] R. L. Jaffe, Phys. Rev. D15, 281 (1977).

[17] H. Högaasen and P. Sorba, Nucl. Phys. B145, 119 (1978).

[18] H. Högaasen and P. Sorba, Mod. Phys. Lett. A19, 2403 (2004); Fizika B14, 245 (2005). 